\title{
Prophylactic Tranexamic acid usage in Prevention of Post-Partum Hemorrhage a Prospective Cohort Study
}

Seifeldin Sadek ( $\square$ ses2020@qatar-med.cornell.edu )

Eastern Virginia Medical School https://orcid.org/0000-0003-0445-4467

Arnold M Mahesan

Eastern Virginia Medical School

Hadi Ramadan

Eastern Virginia Medical School

Nimra Dad

Flushing Hospital Medical Center

Vani Movva

Flushing Hospital Medical Center

Camille Kanaan

Eastern Virginia Medical School

Research article

Keywords: Tranexamic Acid, Post-partum Hemorrhage, Prophylactic, obstetrics

Posted Date: September 24th, 2019

DOI: https://doi.org/10.21203/rs.2.14870/v1

License: (9) (i) This work is licensed under a Creative Commons Attribution 4.0 International License.

Read Full License 


\section{Abstract}

Background Post-partum hemorrhage (PPH) is the leading cause of obstetric morbidity and mortality around the world. Prophylactic administration of tranexamic acid (TXA) in patients at risk for PPH is aimed at reducing estimated blood loss (EBL).

Method This was a prospective cohort study. Patients at high risk of PPH were given 1000mg of TXA intravenously at cord clamping after delivery of the baby, and compared with high PPH risk controls who did not receive TXA. Both cesarean section deliveries (CD) and vaginal deliveries (VD) were included. The primary outcome was postpartum hemorrhage, and the secondary outcome was calculated estimated blood loss (cEBL).

Results Between January 2017 and May 2017, 101 patients at high risk for PPH were enrolled. Fifty one received TXA and 50 did not. There were 20 cases of PPH in the TXA group compared with 30 cases in the control group (OR $0.43,95 \% \mathrm{Cl}, \mathrm{NNT}=5$ ). The average cEBL for VD and CD were significantly lower in the patients who received TXA compared with controls: $561 \mathrm{ml} \pm 336 \mathrm{ml}$ compared with $841 \mathrm{ml} \pm 462 \mathrm{ml}$ $(p=0.0006)$ for VD and $658 \mathrm{ml} \pm 294 \mathrm{~mL}$ compared with $1024 \mathrm{ml} \pm 276$ for $\mathrm{CD}(p=0.0007)$.

Conclusions Intravenous TXA may be used as prophylaxis for PPH in obstetric patients at high risk.

\section{Background}

Post-partum hemorrhage (PPH) is the leading cause of obstetric morbidity and mortality around the world leading to an estimated 140,000 deaths per year $(1,2)$. PPH is defined as the loss of greater than $500 \mathrm{ml}$ of blood after a vaginal delivery or $1000 \mathrm{ml}$ of blood after a c-section delivery(3). The most common causes of PPH are uterine atony, which accounts for nearly $70 \%$ of all cases, and genital tract trauma. Less common causes include retained placenta, coagulation disorders, uterine inversion, and placenta accreta/previa.

Tranexamic acid (TXA) has been shown to decrease blood loss both perioperatively as well as postoperatively(4)'(5).TXA is a synthetic derivative of the amino acid lysine. It inhibits fibrinolysis by blocking the lysine binding site of plasminogen to fibrin $(6,7)$. This antifibrinolytic mechanism has been shown to reduce blood loss in various clinical settings. There have been few randomized control trials assessing tranexamic acid its efficacy at reducing adverse outcomes associated with PPH (8-10).

Recently, TXA was used in the WOMAN trial to prove that if given within 3 hours of diagnosing PPH, it significantly reduced morbidity and mortality(11).

Following these findings, the American College of Obstetricians and Gynecologists (ACOG), released a commentary stating that intravenous tranexamic acid is an second-line therapeutic option for the treatment of PPH (3). 
A systematic review suggests that tranexamic acid decreases post-partum blood loss after both vaginal delivery (VD) and Cesarean delivery (CD) when given prophylactically $(10,12)$

Recently, TXA was used in the WOMAN trial to prove that if given within 3 hours of diagnosing PPH, it significantly reduced morbidity and mortality(11).

Following these findings, the American College of Obstetricians and Gynecologists (ACOG), released a commentary stating that intravenous tranexamic acid is an second-line therapeutic option for the treatment of PPH (3).

Sentilhes et al explored the utility of TXA for prevention of PPH. They found that administering $1 \mathrm{~g}$ of intravenous (IV) TXA to women undergoing vaginal delivery did not result in a decrease in the rate of $\mathrm{PPH}$. However, this study included both high risk and low risk women.

The aim of our study was to determine the effectiveness of prophylactic IV TXA use to decrease estimated blood loss (EBL) in women at medium to high risk undergoing VD or CD. We hypothesized that the use of prophylactic IV TXA would decrease PPH as a secondary outcome.

\section{Methods}

Our study was a single-center prospective cohort study. Institutional Review Board approval was obtained (Protocol \#04/16-2). Using the ACOG PPH risk assessment tool, patients who were deemed medium or high risk for PPH were identified (13). Over a 6-month period, women at medium or high risk for PPH who were at least 32 weeks in gestation, age $>18$ years, and planning to deliver vaginally or via CD were included in the study. Patients who had severe existing medical complications involving the heart, liver, or kidney, known hemostatic abnormalities before pregnancy, or age $<18$, were not included in the study.

Patients meeting inclusion criteria were approached during their admission to labor and delivery. They were assigned to either treatment group or control randomly. Patient who did not desire to be in the treatment group were still approached to be involved as controls.

Roughly half of the women in the study were given TXA $1 \mathrm{~g}$ IV over 3-10 minutes immediately after cord clamping. The other half did not receive TXA. Twenty units of IV Pitocin was administered as standard protocol for all deliveries. Further management of PPH was at the discretion of the delivering provider.

The primary outcome was calculated estimated blood loss (cEBL), it was derived by multiplying the calculated maternal blood volume by the percent of blood volume lost according to the below equations (14).

Maternal blood volume $=0.75 \times[($ maternal height in inches X 50) $+($ maternal weight in pounds +25$)]$

Percent of blood volume lost $=($ predelivery HCT - postdelivery HCT $) /$ predelivery HCT 
Secondary outcomes included post-partum hemorrhage (defined as $\geq 500 \mathrm{ml}$ for vaginal deliveries and $\geq 1000 \mathrm{ml}$ for c-section), duration of IV fluid administration in minutes, total number of uterotonics given, hysterectomies performed, and number of units of packed red blood cells (PRBC) transfused. Side effects such as venous thromboembolism, nausea, vomiting, and dizziness were also studied.

Statistical Analysis:

To detect a $300 \mathrm{ml}$ difference in blood loss at $90 \%$ power, the total population size was calculated to be 36.

Categorical outcomes of interest were compared using chi square test and continuous variables were compared using Student t-test. A logistic regression was performed to adjust for potential confounders, and adjusted odds ratios (AOR) with $95 \%$ confidence intervals were reported. P values less than 0.05 were considered significant.

\section{Results}

Between January 2017 and May 2017, 101 women were enrolled in the study. Fifty-one patients received tranexamic acid $(n=51)$ and fifty patients were used as controls $(n=50)$. The baseline characteristics were similar between the treatment groups (Table 1.), which includes age, gestational age (GA), parity, race, and $\mathrm{BMI}$.

There was a total of fifty (49.5\%) cases of PP; of those, twenty (40\%) women received TXA, while thirty $(60 \%)$ women did not. The average CEBL for VD who received TXA was significantly less than for those who did not $(561 \mathrm{ml} \pm 336$ vs $841 \mathrm{ml} \pm 462, p=0.0006)$. The average cEBL for CD who received TXA was also significantly less than for those who did not $(658 \mathrm{ml} \pm 294 \mathrm{vs} 1024 \mathrm{ml} \pm 276, p=0.0007)$. The duration of IV fluid administration did not significantly differ between treatment and control groups (415 min vs $470 \min \mathrm{p}=0.58$ )

Only one woman on each arm underwent emergent hysterectomy; both patients had placenta accreta. Five women who received TXA required extra uterotonics, compared with eight who did not receive TXA; this difference was not significant $(p=0.18)$. A significantly greater number of pRBCs was transfused in women who did not receive TXA ( 4 vs $0, p=0.02$ ). There were no reported cases of pulmonary embolism or deep vein thrombosis in either group.

Logistic regression models were created to predict PPH in patients receiving TXA. Quality of data fit was assessed by Hosmer and Lemeshow test. Significantly fewer patients who received TXA had PPH, Odds Ratio (OR) $0.43,95 \% \mathrm{Cl}$, with number needed to treat being 5 women. The AOR of PPH in patients receiving TXA was $0.46,95 \% \mathrm{Cl} 1.09-7.67$. Age, BMI, race, parity, GA were all not significant (Table 3 )

\section{Discussion}


We found a significant reduction in $\mathrm{CEBL}$ in both VD and CD in comparison to the controls and when we performed a logistic regression to control for independent variables, women that received TXA prophylactically had a 2.89 times reduction in $\mathrm{PPH}$. Notably, none of the patients receiving prophylactic TXA required blood products; this has the potential to accrue a significant cost and risk reduction for hospitals and labor and delivery units.

There have been few studies on the prophylactic administration of tranexamic acid to prevent postpartum hemorrhage. (15). According to the WOMAN trial when TXA is administered within 3 hours of diagnosis of post-partum hemorrhage, there was a significant reduction in blood loss $(16,17)$. Leading to the notion of earlier administration of TXA and even prophylactic administration.

A recent systematic review and Cochrane review showed that prophylactic administration of TXA reduced blood loss in low and high-risk deliveries. However, the data was lacking in quality, and the available studies predominantly included low-risk patients $(12,18)$.

Recent completion of the TRAAP trial, Sentilhes et al showed that women undergoing VD who received prophylactic tranexamic acid did not result in the reduction in the rate of estimated blood loss in comparison to placebo(15). One limitation of the was the inclusion of both low and high risk women for $\mathrm{PPH}$, which

We believe the main strength of our study in comparison to previous studies on prophylactic TXA, is our inclusion of moderate to high-risk women only. We believe when TXA was given prophylactically it significantly reduced the $\mathrm{cEBL}$ and the rate of $\mathrm{PPH}$ in that patient population.

Currently the ACOG guidelines on TXA usage for post-partum hemorrhage, state that TXA should only be used if uterotonics fail to stop the bleeding or if it is thought that the bleeding may be due to trauma $(3,19)$. We believe that TXA should be used prophylactically in select patient populations that are at higher risk for $\mathrm{PPH}$.

Our study had several strengths but some limitations. Even though most of our results were both statistically and clinically significant, a larger population is required to evaluate for adverse events related to TXA administration. We were unable to elicit any side effects associated with TXA administration. However, in previous studies Sentilhes et al and Shakur et al showed no significant increase in the number of serious side effects such as DVT or PE.

Another minor limitation was our inability to control the amount of intravenous fluids the patients received. This could theoretically cause hemodilution affecting the results of the cEBL. However, we assessed the total duration of IV fluid administration and found no significant difference between treatment and control groups.

Finally, given the inherent nature of our study, another potential limitation is physician bias. The knowledge of TXA administration could potentially affect physician management of PPH. Since there 
was no significant difference between number of uterotonics given, we believe that bias to have been minimal.

\section{Conclusion}

Tranexamic acid, when administered prophylactically, can significantly reduce blood loss in women at increased risk for PPH. Women who received TXA in this study showed a significant reduction in blood loss and post-partum hemorrhage, in both cesarean sections and vaginal deliveries.

Considering our results, a randomized controlled trial is warranted to further assess the use of prophylactic TXA in prevention of PPH in high-risk women. Our data suggest there may be significant benefit to prophylactic use of TXA.

\section{Abbreviations}

Post-partum hemorrhage (PPH)

Tranexamic acid (TXA)

Estimated blood loss (EBL)

Cesarean section deliveries (CD)

Vaginal deliveries (VD)

Calculated estimated blood loss (cEBL)

American College of Obstetricians and Gynecologists (ACOG)

Intravenous (IV)

Packed red blood cells (PRBC)

Gestational age (GA)

Adjusted Odds ratios (AOR)

Odds ratios (OR)

\section{Declarations}

- Ethics approval and consent to participate

Approval for human subjects research was obtained via the Flushing Hospital Medical Center IRB committee; (Protocol \#04/16-2). 
- Consent for publication

Not applicable

$\triangle$ Availability of data and material

Data and Material is submitted as Supplement 1

$\nabla$ Competing interests

The authors declare that they have no competing interests

\ Funding

No funding was used for this study

Q Authors' contributions

Seifeldin Sadek:

Protocol/project development

Data collection or management

Data analysis

Manuscript writing/editing

Arnold M Mahesan:

Data analysis

Manuscript writing/editing

Hadi Ramadan:

Manuscript writing/editing

Nimra Dad:

Data collection or management

Manuscript writing/editing

Vani Movva:

Data collection or management 
Manuscript writing/editing

Camille Kanaan:

Manuscript writing/editing

\Acknowledgements

Not Applicable

- Endnotes

Not Applicable

\section{References}

1. AbouZahr C. Global burden of maternal death and disability. Br Med Bull. 2003;67:1-11.

2. Abouzahr C, Wardlaw T. Maternal Mortality in 2000: Estimates developed by WHO, UNICEF, UNFPA. Who. 2004;1-30.

3. ACOG Practice Bulletin. Clinical Management Guidelines for Obstetrician Gynecologists Number 76: postpartum haemorrhage. J Obstet Gynaecol (Lahore). 2006;108(4):1039-47.

4. Reid RW, Zimmerman AA, Laussen PC, Mayer JE, Gorlin JB, Burrows FA. The efficacy of tranexamic acid versus placebo in decreasing blood loss in pediatric patients undergoing repeat cardiac surgery. Anesth Analg. 1997 May;84(5):990-6.

5. Dryden PJ, O'Connor JP, Jamieson WR, Reid I, Ansley D, Sadeghi H, et al. Tranexamic acid reduces blood loss and transfusion in reoperative cardiac surgery. Can J Anaesth. 1997 Sep;44(9):934-41.

6. Astedt B. Clinical pharmacology of tranexamic acid. Scand J Gastroenterol Suppl. 1987;137:22-5.

7. Longstaff $C$. Studies on the mechanisms of action of aprotinin and tranexamic acid as plasmin inhibitors and antifibrinolytic agents. Blood Coagul Fibrinolysis. 1994 Aug;5(4):537-42.

8. Gai M, Wu L, Su Q, Tatsumoto K. Clinical observation of blood loss reduced by tranexamic acid during and after caesarian section: a multi-center, randomized trial. Eur J Obstet Gynecol Reprod Biol. 2004 Feb;112(2):154-7.

9. Senturk MB, Cakmak Y, Yildiz G, Yildiz P. Tranexamic acid for cesarean section: a double-blind, placebo-controlled, randomized clinical trial. Arch Gynecol Obstet. 2013 Apr;287(4):641-5.

10. Novikova N, Hofmeyr GJ. Tranexamic acid for preventing postpartum haemorrhage. Cochrane database Syst Rev. 2010 Jul;(7):CD007872.

11. Li B, Miners A, Shakur H, Roberts I. Tranexamic acid for treatment of women with post-partum haemorrhage in Nigeria and Pakistan: a cost-effectiveness analysis of data from the WOMAN trial. Lancet Glob Heal. 2018 Feb;6(2):e222-8. 
12. Novikova N, Gj H, Cluver C. Tranexamic acid for preventing postpartum haemorrhage (Review) SUMMARY OF FINDINGS FOR THE MAIN COMPARISON. 2015;(6).

13. Practice Bulletin No. 183: Postpartum Hemorrhage. Obstet Gynecol. 2017 Oct;130(4):e168-86.

14. Stafford I, Dildy GA, Clark SL, Belfort MA. Visually estimated and calculated blood loss in vaginal and cesarean delivery. Am J Obstet Gynecol. 2008;199(5):1-7.

15. Sentilhes L, Winer N, Azria E, Senat M-V, Le Ray C, Vardon D, et al. Tranexamic Acid for the Prevention of Blood Loss after Vaginal Delivery. N Engl J Med. 2018 Aug;379(8):731-42.

16. Shakur H, Roberts I, Fawole B, Chaudhri R, El-Sheikh M, Akintan A, et al. Effect of early tranexamic acid administration on mortality, hysterectomy, and other morbidities in women with post-partum haemorrhage (WOMAN): an international, randomised, double-blind, placebo-controlled trial. Lancet. 2017;389(10084):2105-16.

17. Moore EE, Moore HB, Gonzalez E, Chapman MP, Hansen KC, Sauaia A, et al. Postinjury fibrinolysis shutdown: Rationale for selective tranexamic acid. J Trauma Acute Care Surg. 2015;78(6):S65-9.

18. Li C, Gong Y, Dong L, Xie B, Dai Z. Is prophylactic tranexamic acid administration effective and safe for postpartum hemorrhage prevention? A systematic review and meta-analysis. Medicine (Baltimore) [Internet]. 2017;96(1):e5653. Available from: http://content.wkhealth.com/linkback/openurl?sid = WKPTLP:landingpage\&an = 00005792201701060-00020

19. Pacheco LD, Hankins GD V, Saad AF, Costantine MM, Chiossi G, Saade GR. Tranexamic Acid for the Management of Obstetric Hemorrhage. Obstet Gynecol. 2017 Oct;130(4):765-9.

\section{Tables}

Table 1. Demographics. C-section (CD). Normal Vaginal Delivery (VD). Tranexamic Acid (TXA). Gestational Age (GA). Packed Red Blood Cells (PRBC's). 


\begin{tabular}{|l|l|l|l|}
\hline & TXA (n) & No-TXA (n) & P value \\
\hline Number & 51 & 50 & \\
\hline VD & 31 & 29 & \\
\hline CD & 20 & 21 & \\
\hline Average duration of fluid hydration & 415 & 470 & $\mathrm{p}=0.58$ \\
\hline Race (\%) & & & \\
\hline Hispanic & 59 & 58 & \\
\hline Asian & 37 & 36 & \\
\hline Black & 0 & 2 & \\
\hline Other & 4 & 4 & \\
\hline Age & $35.6 \pm 4.3$ & $37 \pm 3.5$ & $\mathrm{p}=0.06$ \\
\hline BMI & $29.5 \pm 5.4$ & $31.5 \pm 4.7$ & $\mathrm{p}=0.06$ \\
\hline Parity & $3 \pm 1.3$ & $3 \pm 1.3$ & $\mathrm{p}=0.13$ \\
\hline GA (weeks) & $383 / 7 \pm 2.3$ & $383 / 7 \pm 2.4$ & $\mathrm{p}=0.88$ \\
\hline
\end{tabular}

Table 2. Outcomes. C-section (CD). Normal Vaginal Delivery (VD). Tranexamic Acid (TXA). Estimated Blood loss (EBL). Gestational Age (GA). Packed Red Blood Cells (PRBC's). Post-partum Hemorrhage (PPH). Not available (N/A)

\begin{tabular}{|l|c|c|c|}
\hline & TXA & No-TXA & p-value \\
\hline Total EBL(ml) & $599 \pm 318$ & $918 \pm 394$ & 0.00003 \\
\hline VD EBL(ml) & $561 \pm 336$ & $841 \pm 454$ & 0.0006 \\
\hline CD EBL(ml) & $658 \pm 287$ & $1024 \pm 270$ & 0.0007 \\
\hline Uterotonics (n) & 5 & 8 & 0.18 \\
\hline PRBC's(units) & 0 & 4 & 0.02 \\
\hline Hysterectomy (n) & 1 & 1 & 0.16 \\
\hline PPH (n) & 20 & 31 & 0.03 \\
\hline Side effects & 0 & 0 & N/A \\
\hline
\end{tabular}

Table 3. Odds Ratio of TXA, *Adjusted for age, BMI, race, parity and GA 


\begin{tabular}{|l|l|l|}
\hline & TXA ppx Odds ratio, (OR) 95\% Cl & TXA ppx Adjusted Odds ratio (AOR)* 95\% Cl \\
\hline PPH & $0.43(p=0.37)$ & $0.46(p=0.32)$ \\
\hline
\end{tabular}

\section{Figures}

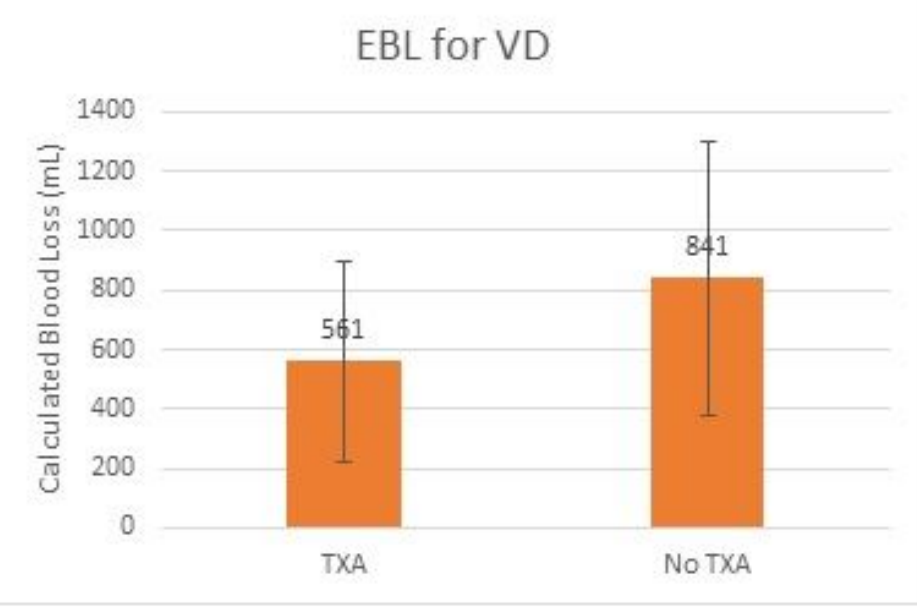

A

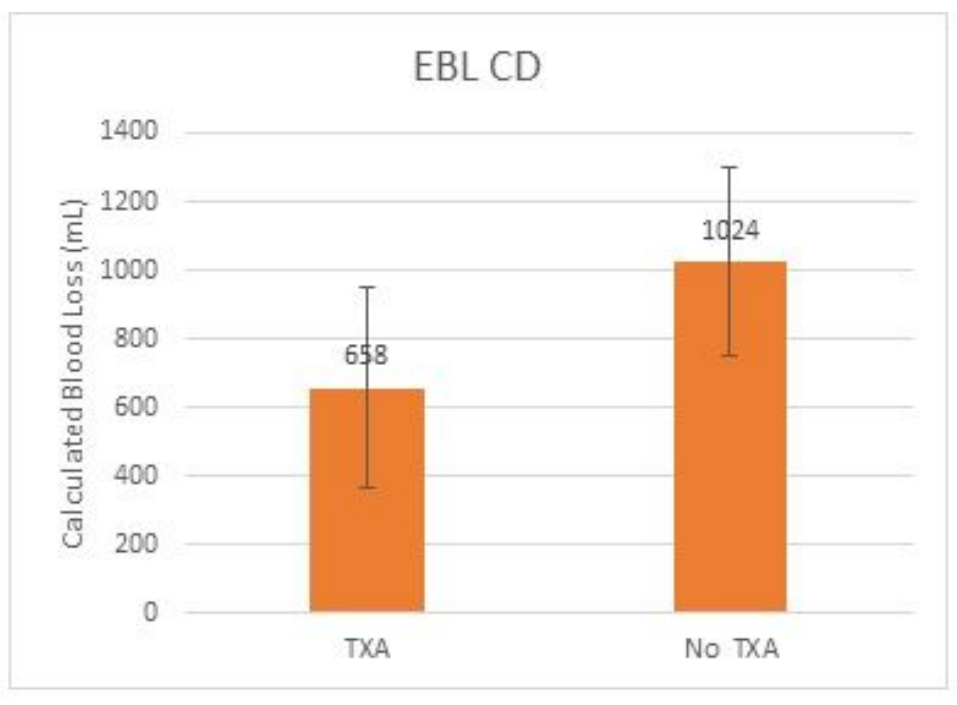

B

Figure 1

A. Calculated blood loss for patients with Vaginal Delivery (VD), B Calculated blood loss for patients with c-section (CD). Tranexamic Acid (TXA). Estimated Blood Loss (EBL).

\section{Supplementary Files}

This is a list of supplementary files associated with this preprint. Click to download.

- STROBEchecklistv4combined.doc

- supplementaldata.xlsx 